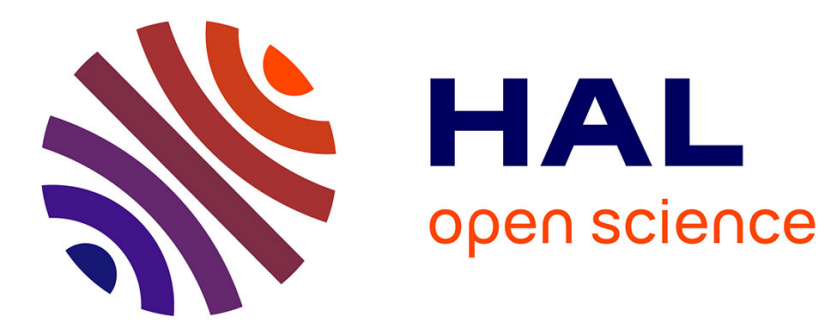

\title{
Architecture level Optimizations for Kummer based HECC on FPGAs
}

\author{
Gabriel Gallin, Turku Ozlum Celik, Arnaud Tisserand
}

\section{To cite this version:}

Gabriel Gallin, Turku Ozlum Celik, Arnaud Tisserand. Architecture level Optimizations for Kummer based HECC on FPGAs. IndoCrypt 2017 - 18th International Conference on Cryptology in India, Dec 2017, Chennai, India. pp.44-64, 10.1007/978-3-319-71667-1_3 . hal-01614063

\section{HAL Id: hal-01614063 https://hal.science/hal-01614063}

Submitted on 10 Oct 2017

HAL is a multi-disciplinary open access archive for the deposit and dissemination of scientific research documents, whether they are published or not. The documents may come from teaching and research institutions in France or abroad, or from public or private research centers.
L'archive ouverte pluridisciplinaire HAL, est destinée au dépôt et à la diffusion de documents scientifiques de niveau recherche, publiés ou non, émanant des établissements d'enseignement et de recherche français ou étrangers, des laboratoires publics ou privés. 


\title{
Architecture level Optimizations for Kummer based HECC on FPGAs
}

\author{
Gabriel GALLIN ${ }^{1}$, Turku Ozlum CELIK ${ }^{2}$, and \\ Arnaud TISSERAND ${ }^{3[0000-0001-7042-3541]}$ \\ ${ }^{1}$ CNRS, IRISA UMR 6074, INRIA Centre Rennes - Bretagne Atlantique and \\ University Rennes 1, Lannion, France \\ gabriel.gallin@irisa.fr \\ 2 IRMAR, University Rennes 1, Rennes, France \\ turku-ozlum.celik@univ-rennes1.fr \\ 3 CNRS, Lab-STICC UMR 6285 and University South Britany, Lorient, France \\ arnaud.tisserand@univ-ubs.fr
}

\begin{abstract}
On the basis of a software implementation of Kummer based HECC over $\mathbb{F}_{p}$ presented in 2016, we propose new hardware architectures. Our main objectives are: definition of architecture parameters (type, size and number of units for arithmetic operations, memory and internal communications); architecture style optimization to exploit internal parallelism. Several architectures have been designed and implemented on FPGAs for scalar multiplication acceleration in embedded systems. Our results show significant area reduction for similar computation time than best state of the art hardware implementations of curve based solutions.
\end{abstract}

Keywords: hyper-elliptic curve cryptography, hardware implementation, architecture exploration, embedded systems.

\section{Introduction}

Reducing the cost of asymmetric cryptography is a challenge for hardware implementation of embedded systems where silicon area is limited. Hyper-elliptic curve cryptography (HECC [6]) is considered to be an interesting solution compared to elliptic curve cryptography (ECC [12]). HECC requires smaller finite fields than ECC at similar security level. For instance, size of field elements is divided by two in genus-2 HECC solutions. But the number of field-level operations is larger in HECC per key/scalar bit. Then comparisons depend a lot on curve parameters, algorithm optimizations and implementation efforts.

HECC solutions based on Kummer surfaces (see [10] for details) demonstrate promising improvements for embedded software implementations. In 2016, Renes et al. presented in 24 a new Kummer-based HECC (KHECC) solution and its implementation on microcontrollers with 30 to $70 \%$ clock cycles count reduction compared to the best similar curve based solutions at equivalent security level.

To the best of our knowledge, there is no hardware implementation of this recent KHECC solution. Below, we present hardware architectures for KHECC 
adapted from 24] for scalar multiplication and their FPGA implementations. We study and evaluate the impact of various architecture parameters on the cost and performances: type, size and number of units (arithmetic, memory, internal communications); architecture topology; and exploitation of internal parallelism. We target embedded applications where the FPGA bitstream cannot be changed easily (trusting the configuration system at application level is complex) or prototyping for ASIC applications. Then to provide flexible circuits at software level, our solutions are designed for $\mathbb{F}_{p}$ with generic primes (where [24] only deals with $p=2^{127}-1$ ). Several architectures have been designed and implemented on different FPGAs for various parameters, and compared in terms of area and computation time.

Our paper outline is as follows. Section 2 recalls background on KHECC and introduces notations. Section 3 presents major elements of the work [24] used as a starting point and discusses required adaptations for hardware implementation. Section 4 quickly presents our units selected for the architectures and our tools used for design space exploration. Section 5 describes the proposed KHECC architectures and their implementation results on different FPGAs. Section 6 reports comparisons. Finally, Section 7 concludes the paper.

\section{State-of-the-Art}

HECC was introduced by Koblitz in 14] as a larger set of curves compared to ECC with a generalization of the class of groups obtained from the jacobians of hyper-elliptic curves. Subsequently, many HECC improvements have been proposed. See book [6] for a complete presentation on HECC and book [12] for ECC. Broadly speaking, field elements in HECC are smaller than in ECC for a similar security level (e.g. 128-bit HECC on genus-2 curves is equivalent to 256-bit ECC). This reduction should directly benefit to HECC since the width of field elements has a major impact on circuit area. But HECC requires more field operations to achieve operations at curve level such as point addition (ADD) and point doubling (DBL) for each scalar/key bit.

Many efforts have been made to reduce the cost of curve level operations in HECC. For genus-2, one can refer to Lange [18, Gaudry [10], Bos et al. [5] and Renes et al. 24] for instance. Table 1 reports a few costs for HECC and ECC solutions. There were many works on $\mathbb{F}_{2^{n}}$ solutions at low security levels (fields with $80-90$ bits) in the past but very few on $\mathbb{F}_{p}$ at 128 -bit security level until recently in software (our goal is hardware implementation).

KHECC solutions from [24] are based on a Kummer surface $\mathcal{K}_{\mathcal{C}}$ of an hyperelliptic curve $\mathcal{C}$ defined over $\mathbb{F}_{p}$. Curve $\mathcal{C}$ is defined using constant parameters among which the "squared theta constants" $(a, b, c, d)$ used during scalar multiplications. Points are represented by tuples of four $n$-bit coordinates in $\mathbb{F}_{p}$ where $\pm P=\left(x_{P}: y_{P}: z_{P}: t_{P}\right)$ is the projection of $P$ from $\mathcal{C}$ on $\mathcal{K}_{\mathcal{C}}$.

In $(\mathrm{H})$ ECC primitives such as signature or key exchange, the main operation is the scalar multiplication $[k] P_{b}$ of a base point $P_{b}$ by a $m$-bit scalar or key $k$. In embedded systems, scalar multiplication must be protected against side channel 
attacks (SCAs [20]). A popular protection against SCAs is the adaptation of Montgomery ladder (ML) algorithm [22. ML is constant time (i.e. computation time of iterations does not depend on the key bit values) and uniform (i.e. the exact same schedule of the exact same field operations is executed at each iteration whatever the key bit values).

For KHECC hardware implementations (but also for more general HECC solutions), designers have to face several questions. How one should exploit the internal parallelism available at field level? Are few large and fast units more efficient than several parallel small and slow units? How to select parameters in a parallel architecture? Our work was related to those questions.

\section{Hardware Adaptation of Renes et al. Solution}

We based our work on the KHECC solution presented by Renes et al. at CHES 2016 [24] for software implementations of Diffie-Hellman key exchange and signature at 128-bit security level. Their solution optimizes the use of Kummer surface of hyper-elliptic curve described by Gaudry [10].

\subsection{Analysis of Renes et al. Solution}

In [24, the prime for $\mathbb{F}_{p}$ is $p=2^{127}-1$ due to fast modular reduction algorithms for Mersenne primes. The scalar size is $m=256$ bits. ML algorithm starts with most significant key bits first. Each iteration computes a couple of points $\left( \pm V_{1}, \pm V_{2}\right)$ of $\mathcal{K}_{\mathcal{C}}$ from the result of the previous iteration using curve level operations CSWAP and XDBLADD. Using initial values $\pm V_{1}=(a: b: c: d)$ and $\pm V_{2}=$ $\left(x_{P_{b}}: y_{P_{b}}: z_{P_{b}}: t_{P_{b}}\right)$, the scalar multiplication computes $\left( \pm[k] P_{b}, \pm[k+1] P_{b}\right)$.

The core operation in ML iterations is the modified pseudo-addition xDBLADD combined differential double-and-add (see [24]). Given points $\pm V_{1}, \pm V_{2}$ on $\mathcal{K}_{\mathcal{C}}$, and base point $\pm P_{b}$, it computes $\left( \pm[2] V_{1}, \pm\left(V_{1}+V_{2}\right)\right)=\operatorname{xDBLADD}\left( \pm V_{1}, \pm V_{2}, \pm P_{b}\right)$. Based on the set of $\mathbb{F}_{p}$ operations described in Figure 1 , xDBLADD has a constant time and uniform behavior.

The CSWAP operation consists in swapping the 2 input points (IN) of xDBLADD and the 2 resulting points (OUT) depending on the current key bit value (see Algo. 7 in 24). It does not involve any computation but it impacts SCA aspects.

Renes et al. performed a smart selection of optimized curve parameters (from [10]) to determine constants with reduced size: 16 bits instead of 127 .

\begin{tabular}{|c||c|c|c|}
\hline solution \& source & field width [bit] & ADD & DBL \\
\hline \hline $\mathbb{F}_{p}$ ECC $[4]$ & $\ell_{\mathrm{ECC}}$ & $12 \mathrm{M}+2 \mathrm{~S}$ & $7 \mathrm{M}+3 \mathrm{~S}$ \\
\hline $\mathbb{F}_{2^{n}}$ HECC $[18]$ & $\ell_{\mathrm{HECC}} \approx 0.5 \ell_{\mathrm{ECC}}$ & $40 \mathrm{M}+4 \mathrm{~S}$ & $38 \mathrm{M}+6 \mathrm{~S}$ \\
\hline $\mathbb{F}_{p}$ KHECC $[24]$ & $\ell_{\mathrm{HECC}} \approx 0.5 \ell_{\mathrm{ECC}}$ & \multicolumn{2}{|c|}{$19 \mathrm{M}+12 \mathrm{~S}$} \\
\hline
\end{tabular}

Table 1. Cost per key bit of curve level operations in various (H)ECC solutions (M and $\mathbf{S}$ denote multiplication and square in the finite field). 
Then they use a dedicated optimized function for modular multiplication by this type of constants in the software implementation.

Their implementations target low-cost microcontrollers: 8-bit AVR AT Mega and 32-bit ARM Cortex M0. They report significant improvements over the best known solutions. On Cortex M0, the clock cycles count is reduced by $27 \%$ for key exchange and by $75 \%$ for scalar multiplication in signature. On AT Mega, the corresponding reductions are respectively $32 \%$ and $71 \%$.

\subsection{Objectives and Constraints for our Hardware Accelerators}

Unlike Renes et al. 24, in the present paper we only propose hardware acceleration for scalar multiplication since this is the main operation in terms of performance, energy consumption and security against SCAs (when the scalar is the private key). As is frequently the case in a complete embedded system, we assume that our hardware accelerator is coupled to a software implementation for high level primitives (which are out of scope of this paper).

In order to design flexible hardware accelerators and to report results in a general case, we target KHECC on generic prime fields. In 24 the selected prime $p=2^{127}-1$ leads to very cheap modular reduction but it is very specific (there is no Mersenne prime for slightly different security levels). Currently, we only deal with generic primes but we plan to derive versions for specific ones (e.g. pseudo-Mersenne) in the future. Field characteristic impacts the choice of curve parameters. We propose to use material presented in the work from Gaudry 10 to derive curves parameters and implementation constants.

One of our goal is to study hardware accelerators for scalar multiplication at architecture level. KHECC offers some internal parallelism as illustrated in Figure 1. Groups of 4 to $8 \mathbb{F}_{p}$ operations can be easily performed at the same time with uniform and constant time schedules. In most of ECC solutions, fewer operations can be performed in parallel. We will evaluate the impact of this parallelism on the design of efficient accelerators with various trade-offs in terms of area and computation time (see questions at end of Section 2).

This paper is not dedicated to protection against physical attacks. But we target hardware accelerators where the execution of ML type of algorithms is actually constant time and uniform at low level. We will describe how we designed some units to achieve this objective (not yet evaluated using real attacks).

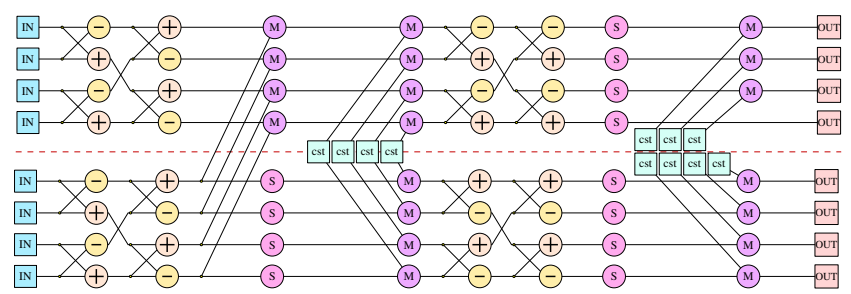

Fig. 1. $\mathbb{F}_{p}$ operations in xDBLADD from $[24]$ (IN/OUT are $\pm V_{1}, \pm V_{2}$ coordinates). 
In order to provide hardware accelerators that can be easily adapted to other application constraints and algorithms, we use a modular type of architectures based on independent units (for arithmetic operations and internal storage); a microcoded control; and an internal communication system based on multiplexors (between some units). This type of modular architectures allows us to easily explore many solutions in design space: type, size and number of units; size of internal communications; scheduling impact on performances and security. This choice also comes from the type of dedicated resources available in modern FPGAs: small embedded multipliers (DSP slices/blocks), small embedded memory (BRAM: block RAM), dedicated multiplexors in routing resources. Architecture modularity also helps the debug and validation process of the proposed solutions using a hierarchical method. First, we extensively and individually simulate in HDL and evaluate on FPGAs all our units. Second, we extensively simulate in HDL and evaluate on FPGAs the complete accelerator solutions.

\section{Accelerator Units and Exploration Tools}

Architecture modularity allows to explore a wide parameter space but it requires huge efforts in terms of implementation and debug. First, we decided to design, implement, validate, and optimize a small set of units for arithmetic operations, memory and internal communications. They are presented in Section 4.1. Second, based on this set of units, we designed specific tools for architecture level exploration and evaluation. These tools are quickly described in Section 4.2.

\subsection{Accelerator Units and Resources}

There are several types of resources in our hardware accelerators:

- arithmetic units for field level operations $\left(\mathbb{F}_{p}\right.$ addition, subtraction and multiplication with generic prime $p$ in this work);

- memory unit(s) for storing intermediate values $\left(\mathbb{F}_{p}\right.$ elements for points coordinates), curve parameters and constants;

- a CSWAP unit in charge of scalar management and on-line schedule of operands depending on scalar/key bit values;

- an internal communication system for data transfers between the units;

- a control based on a microcode running the architecture.

Multiplier (Mult): Prime $p$ is generic and can be programmed in our architecture. Then, there is no low-cost modular reduction. We use the Montgomery modular multiplication (MMM) proposed in 21. Operands and product are represented in Montgomery domain (MD) $\left\{(x \times R) \bmod p, \forall x \in \mathbb{F}_{p}\right\}$ with $\operatorname{gcd}(R, p)=1$ and $R>p$. We use MMM optimization methods from Orup 23 . and Koc et al. [15] where internal high-radix computations reduce the amount of data dependencies and partial product/reduction steps are interleaved to speed up the multiplication with a slightly larger internal datapath. 
In our target Xilinx FPGAs (see Section 5), there are embedded multipliers for $18 \times 18$ bits signed integers called DSP blocks/slices (in 2's complement representation). But for $\mathbb{F}_{p}$ computations, one can only use the 17 LSBs (all but the "sign" bit). Then our datapath width must be a multiple of 17 . We evaluated that 34-bit word size for multiplication is interesting in our KHECC context (smaller size leads to slow multipliers, larger size requires too many DSP slices). For 34-bit words, operands and products are between 0 and $2 p$ represented using 136 bits in MD (136 is the closest multiple of 17 and 34 larger than 127). Then we can select $R>4 p$ for speed purpose (as many works in state of the art).

For improving the efficiency of our accelerators, we used the hyper-threaded multiplier version we proposed in 9] specially for KHECC. Hyper-threading hides the latency in DSP slices at high frequency (when all internal registers are activated). It computes 3 independent MMMs in parallel using 11 DSP slices $(17 \times 17), 2$ BRAMs and a few slices in 79 clock cycles at $360 \mathrm{MHz}$ on Virtex 5 .

Due to MD, all field elements, parameters and constants are 128-bit values (contrary to 24] where shorter constants can be used). Using shorter constants in hardware requires a specific unit and then a more complex control and smaller overall frequency. Also for flexibility and frequency reasons, we only use generic $\mathbb{F}_{p}$ multiplier Mult. For the same reasons, we avoid dedicated square units (for generic $p$, MMM variants for square operation only lead to small improvements).

Similarly to state of the art solutions, final reduction from MD $(0 \leq x<2 p)$ to "standard" $\mathbb{F}_{p}(0 \leq x<p)$ is performed after the scalar multiplication (there is no performance or security issue for this conversion).

Adder (AddSub): It performs both modular addition/subtraction $((x \pm y) \bmod$ $2 p$ ) in MD by setting a mode signal at operation start-up. Subtraction is implemented by adding operand $x$ with the 2's complement of $y$ operand $(\bar{y})$. The reduced sum in $\mathrm{MD}$ (resp. difference), is obtained from the parallel computations $r=x+y$ (resp. $r=x+\bar{y}$ ) and $r_{p}=r+\overline{2 p}$ (resp. $r_{p}=r+2 p$ ). The output is $r$ if $0 \leq r<2 p$, else $r_{p}$. The range of $r$ is determined from the value of the output carry bit of $r+\overline{2 p}$ for addition and of $x+\bar{y}$ for subtraction. We evaluated the impact of AddSub units for several word sizes: 34, 68 and 136 bits. The two large ones significantly reduce the overall frequency of the accelerator (due to longer carry propagations). It seems that our target FPGAs are optimized to handle word sizes around 32 bits but not for larger widths without costly pipeline schemes. To enforce short combinatorial paths and simplify the control, we set AddSub internal datapath width to $w_{\text {arith }}=34$ bits as in Mult.

Memory: The accelerator uses internal memory(ies) for storing intermediate values $\left(\mathbb{F}_{p}\right.$ elements of points coordinates), curve parameters and some constants (e.g. initial values $(a: b: c: d)$ ). To fit the internal width of arithmetic units $\left(w_{\text {arith }}=34\right.$ bits) and BRAM width in Xilinx FPGAs (configurable into $\{1,2,4,9,18,36\}$ bits), we selected a memory configuration where words are multiple of 34 bits to simplify the control and avoid interfaces. Due to the large number of memory operations (read/write), we will show in Section 5 that a wider memory reduces the number of clock cycles per memory operation of full 136-bit values. We tested 3 configurations for the memory width (and internal 


\begin{tabular}{|c||c|c|c|c|}
\hline config. & $w$ [bit] & $s$ [word] & cycle(s) / mem. op. & BRAM(s) \\
\hline \hline w34 & 34 & 4 & 4 & 1 \\
\hline w68 & 68 & 2 & 2 & 2 \\
\hline w136 & 136 & 1 & 1 & 4 \\
\hline
\end{tabular}

Table 2. Memory and internal communication width configurations.

communications see below) described in Table 2 . The main parameter $w$ (in bits) is the internal width of memory words (and communications). Related parameter $s$ is the number of words required for storing a complete 136-bit value. Our BRAMs are configured into 512 lines of 36 bits words (2 unused bits per word). Less than 512 words are required for KHECC even in w34 configuration. Table 2 also reports the clock cycles count of each memory operation and the memory area (in BRAMs). For security reason, this internal memory in restricted to the accelerator and cannot be accessed from outside (inputs and outputs are handled by a specific very small unit which is mute during scalar multiplications).

Internal communications: The units are interconnected through a specific internal communication system based on multiplexors (buses are not very efficient in target FPGAs and lead to high capacitances switching which can be a bad point for SCA protection). The communication system will be described in Section 5 In order to explore cost and performance trade-offs, we used configurations from Table 2 for the width in the internal communication system. Different widths for memory and communications requires a very costly control. Then $w$ is shared for memory and communications. But for arithmetic units in this paper, we evaluated that $w_{\text {arith }}=34$ is the best choice for our KHECC accelerators. For w68 and w136 configurations, small serial-parallel interfaces are added in the arithmetic units to handle the width difference with communications.

CSWAP unit: In algorithm 7 from 24] (called crypto_scalarmult), the CSWAP operation manages the scalar/key bits by swapping, or not, points $\pm V_{1}$ and $\pm V_{2}$ at the beginning and end of each ML iteration. We designed a dedicated unit for this purpose. It reads $2 \mathbb{F}_{p}$ elements as inputs (corresponding to one coordinate of $\pm V_{1}$ and $\pm V_{2}$ ) and swaps them, or not, depending on the actual key bit value for the current iteration. At the last iteration, CSWAP triggers a "end of scalar multiplication" signal. In our CSWAP unit, there is no variable addresses or key management in the accelerator control for security reasons (instructions decoding does not depend on secret bits). For SCA protection, our CSWAP unit has been designed to ensure that there is always electrical activity in the pipelined unit, communication system and memory between successive clock cycles even if there is no swapping (read/write operations for one coordinate are interleaved with those of the other coordinates). Our CSWAP unit (pipelined with internal communications and memory) has a constant time and uniform behavior.

In the future, we will investigate the use of advanced scalar recoding schemes on the performances and security against SCAs.

Accelerator control: We defined a tiny ISA (instruction set architecture). Instructions, detailed in Table 3 , are read from the code memory and decoded to 


\begin{tabular}{|c||c|}
\hline instruc. & description \\
\hline \hline read & transfer operands from memory to target unit and start computation \\
\hline write & transfer result from target unit to memory \\
\hline wait & wait for immediate clock cycles \\
\hline nop & no operation (1 clock cycle) \\
\hline jump & change program counter (PC) to immediate code address \\
\hline end & trigger the end of the scalar multiplication \\
\hline
\end{tabular}

Table 3. Instructions set for our accelerators.

provide control signals to/from units, communication system and memory. The user program is stored into a small program memory (one BRAM). This type of control provides flexibility, avoids long synthesis and place\&route processes (during modifications of user programs), and leads to fairly high frequencies on the target FPGAs when using pipelined BRAMs and decoding.

Instructions are 36-bit wide and our KHECC programs fit into one single BRAM (<512 instructions). Instructions contain: 4-bit opcode, 3-bit unit index, 2-bit operation mode, two 9-bit memory addresses and 9-bit immediate value.

We implemented hardware loops, using small finite state machines (FSMs), to handle $s$ cycles during communication and memory operations and duration of wait instruction. Instruction decoding does not handle or depend on the scalar bit values for SCA protection (only the CSWAP unit handles secret bits).

Control resources include a few $w$-bit registers dedicated to external communications and initialization of memory parameters. They are very small and not involved during scalar multiplications, then we do not detail them here.

In the future, we plan to explore other types of control (e.g. distributed or FSM based solutions without microcode) for ASIC implementations.

\subsection{Tools for Exploration and Evaluation at Architecture Level}

Several parameters must be specified at design time for each architecture:

- type and number of units (AddSub, Mult, CSWAP, memory);

- width $w$ for internal memory and communications;

- topology of the architecture.

All units have been fully described in synthesizable VHDL for FPGA implementation (with optimizations for DSP slices and BRAMs). They can also be tested and evaluated using cycle accurate and bit accurate (CABA) simulations. Then the time model at every clock cycle and the hardware cost of each unit are perfectly known (from implementation results).

Fully designing all possible architectures in VHDL is too time consuming. We decided to define and use a hierarchical and heterogeneous method to efficiently explore and validate numerous architectures. 
Each architecture is described and simulated using a high-level model based on a CCABA (critical CABA) specification 4 . The critical cycles at architecture level are clock cycles where there are transitions in the control signals to/from the units and their inputs/outputs. For functional units, this corresponds to operands inputs, operation mode selection, start of computation, end of computation, and results outputs. The purely internal control signals inside the units are not modeled in CCABA (since their behavior is perfectly determined in the VHDL description and does not impact other parts of the accelerator).

A CCABA simulation tool has been developed in Python. Each unit is modeled in Python to specify: a) its mathematical behavior and b) its behavior at critical cycles based on the corresponding VHDL model (e.g. computation duration after start signal). For each unit, we need its complete VHDL and Python CCABA models (both manually written). Our tool allows fast simulations of complete scalar multiplications due to the hierarchical approach.

We also started the development of a tool to automatically schedule arithmetic and memory operations as well as internal communications. Currently, it uses a basic greedy algorithm to first feed the multipliers (due to their longer latency). We plan to improve it in the future. The schedule gives the total clock cycles count of a complete scalar multiplication.

We are able to quickly estimate the area and computation time of various architectures. The estimated area sums up the VHDL results for all units instantiated in the accelerator. The computation time is estimated by the total number of clock cycles multiplied by the slowest unit period. We approximate the impact of the control system (not yet designed in VHDL at this stage of the exploration) based on our experience.

During the exploration, we perform this type of estimation for each accelerator configuration to be evaluated. Then we select the most interesting solutions for full implementation in VHDL, final validation, and accurate comparisons (the corresponding results are presented in Section 5).

Once the accelerator has been fully implemented in VHDL, it is intensively tested using both VHDL simulations and executions on FPGA cards against reference values computed by SAGE mathematical software.

\section{Proposed Architectures}

We explored various configurations for parameters and architectures. We selected 4 architectures summarized in Table 4 and fully implemented them in VHDL on several FPGAs. The corresponding results are reported in sub-sections below. We began with a small and basic architecture A1 embedding the minimum number of units. Then we explored optimizations and more parallel architectures. Architecture A2 uses an optimization of CSWAP unit (V2). Architecture A3 embeds 2 operators for each arithmetic operation $( \pm$ and $\times)$ to reach a higher parallelism

\footnotetext{
${ }^{4}$ Our CCABA model is inspired by Transaction Level Modeling (TLM) with full cycle accuracy for all control signals at the architecture level but not inside the units (when there is no input/output impact).
} 


\begin{tabular}{|r||c|c|c|c|}
\hline \multicolumn{1}{|c||}{ resources } & \multicolumn{5}{c|}{ architectures } \\
\cline { 2 - 5 } & A1 (Sec. 5.1 & A2 (Sec. 5.2 & A3 (Sec. 5.3 ) & A4 (Sec. 5.4) \\
\hline \hline AddSub & 1 & 1 & 2 & 2 \\
\hline Mult & 1 & 1 & 2 & 2 \\
\hline CSWAP & $1 \mathrm{~V} 1$ & $1 \mathrm{~V} 2$ & $1 \mathrm{~V} 2$ & 1 V3 \\
\hline Communication System & 1 & 1 & 1 & 2 \\
\hline Program Memory & 1 & 1 & 1 & 2 with bridge \\
\hline Control & 1 & 1 & 1 & 1 \\
\hline
\end{tabular}

Table 4. Main characteristics of the 4 implemented and evaluated architectures.

\begin{tabular}{|c||c|c|c|c|c|c|}
\hline \multirow{2}{*}{$\begin{array}{c}\text { short } \\
\text { name }\end{array}$} & model & techno. & \multicolumn{2}{|c|}{ slice content } & $f_{\max }$ DSP & BRAM \\
{$[\mathrm{nm}]$} & LUT & flip-flop & {$[\mathrm{MHz}]$} & capacity \\
\hline \hline V4 & Virtex 4 VLX100 & 90 & 2 LUT4 & 2 & 500 & $18 \mathrm{~Kb}$ \\
\hline V5 & Virtex 5 LX110T & 65 & 4 LUT6 & 4 & 550 & $36 \mathrm{~Kb}$ \\
\hline S6 & Spartan 6 SLX75 & 45 & 4 LUT6 & 4 & 390 & $18 \mathrm{~Kb}$ \\
\hline
\end{tabular}

Table 5. Target FPGAs with some characteristics ( $f_{\max }$ is the maximum frequency).

(notice that a single Mult already handles 3 sets of operands in parallel using hyper-threading, see [9]). Architecture A4 is a cluster of parallel units for both arithmetic operations and data memory operations, and V3 of CSWAP unit.

The Xilinx FPGAs listed in Table 5 were our implementation targets. ISE 14.7 tools were used for synthesis and place\&route, as well as SmartXplorer. V4/V5 FPGAs were used for comparison with state of the art. FPGA S6 was used for low-cost solutions and imminent SCA evaluation on SAKURA card [16]. In order to fairly compare area results, it should be remembered that slice and look-up table (LUT) definition strongly depends on the FPGA family, see examples in Table 5. Flip-flop (FF) means a 1-bit register. One LUT6 is equivalent to 4 LUT4. Then slices in V4 or in V5/S6 should not be compared directly.

For architectures A1-4, we report below implementation results for w34, w68, w136 configurations on V4, V5, S6 FPGAs using 100 SmartXplorer runs.

\subsection{Architecture A1: Base Solution}

Architecture A1, depicted in Figure 2, corresponds to a basic Harvard processor dedicated to the scalar multiplication derived from [24] with our modifications detailed in Section 3.2 . This is the smallest accelerator with only one instance of each type of unit (AddSub, Mult, and CSWAP-V1 described in Section 4.1).

Architecture A1 was fully implemented in VHDL for the 3 widths $w \in$ $\{34,68,136\}$ on 3 FPGAs $\{\mathrm{V} 4, \mathrm{~V} 5, \mathrm{~S} 6\}$. The corresponding results are reported in Table 6, Intensive VHDL simulations were used for validation.

Table 6 reveals a few trends:

- Width $w$ has a small impact (at most $5 \%$ reduction) on the clock cycles count since most of the time is spent into the single AddSub and Mult. 


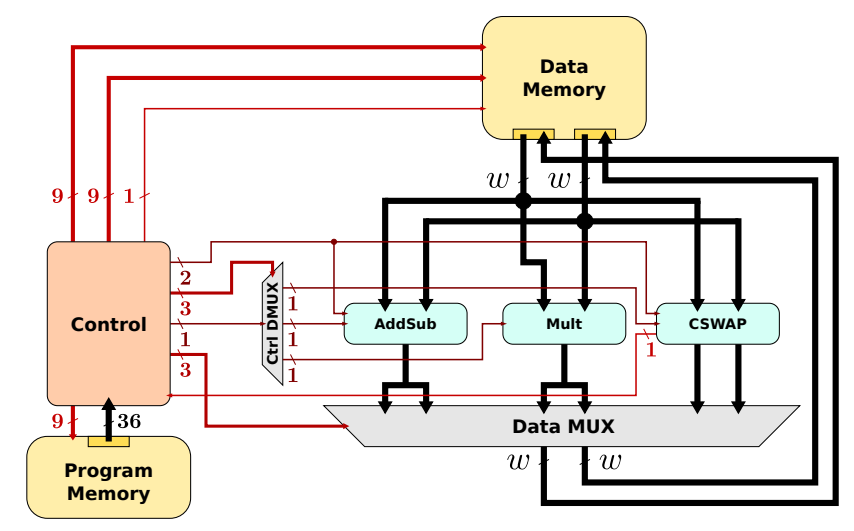

Fig. 2. Architecture A1 with its main units (arithmetic and memory), internal communication system, and control (warning: areas of boxes are not on a real scale).

- Width $w$ strongly impacts the area in LUTs $(+50-70 \%$ for w68 compared to w34, and $+80-110 \%$ for w136 compared to w34 depending on the FPGA). Clearly, enlarging the datapath requires more logic cells.

- The link between $w$ and flip-flops numbers seems tricky. This is partly due to the cost of serial-parallel interfaces between arithmetic operators $\left(w_{\text {arith }}=\right.$ 34 ) and memory/communications (w68 the most complex case, and w136).

- The increase in BRAMs comes from the wider memory for configurations w68 and w136 (see Table 2).

- Frequency decreases when $w$ increases due to longer combinatorial delays and larger fanout. Depending on the FPGA, the reduction varies about 5$20 \%$. On V5, $360 \mathrm{MHz}$ is the frequency for both the slowest unit and the complete accelerator (the control does not impact the overall frequency for a small accelerator which uses a small part of the complete FPGA).

\begin{tabular}{|c|c||c|c|c|c|c|c|c|c|}
\hline FPGA & $\begin{array}{c}w \\
{[\mathrm{bit}]}\end{array}$ & LUT & FF & $\begin{array}{c}\text { logic } \\
\text { slices }\end{array}$ & $\begin{array}{c}\text { DSP } \\
\text { blocks }\end{array}$ & $\begin{array}{c}\text { RAM } \\
\text { blocks }\end{array}$ & $\begin{array}{c}\text { freq. } \\
{[\mathrm{MHz}]}\end{array}$ & $\begin{array}{c}\text { clock } \\
\text { cycles }\end{array}$ & $\begin{array}{c}\text { time } \\
{[\mathrm{ms}]}\end{array}$ \\
\hline \hline \multirow{3}{*}{ V4 } & 34 & 1010 & 1833 & 1361 & 11 & 4 & 322 & 194,614 & 0.60 \\
\cline { 2 - 10 } & 68 & 1750 & 3050 & 2251 & 11 & 5 & 305 & 186,911 & 0.61 \\
\cline { 2 - 10 } & 136 & 2281 & 3028 & 1985 & 11 & 7 & 266 & 184,337 & 0.69 \\
\hline \multirow{3}{*}{ V5 } & 34 & 757 & 1816 & 603 & 11 & 4 & 360 & 194,614 & 0.54 \\
\cline { 2 - 10 } & 68 & 1264 & 3033 & 908 & 11 & 5 & 360 & 186,911 & 0.52 \\
\cline { 2 - 10 } & 136 & 1582 & 3008 & 940 & 11 & 7 & 360 & 184,337 & 0.51 \\
\hline \multirow{3}{*}{ S6 } & 34 & 1064 & 1770 & 408 & 11 & 4 & 278 & 194,614 & 0.70 \\
\cline { 2 - 9 } & 68 & 1555 & 2970 & 705 & 11 & 5 & 252 & 186,911 & 0.74 \\
\cline { 2 - 9 } & 136 & 1910 & 2994 & 747 & 11 & 7 & 221 & 184,337 & 0.83 \\
\hline
\end{tabular}

Table 6. FPGA implementation results for architecture A1 (all BRAMs are $18 \mathrm{~Kb}$ ones, only $17 \times 17$ multipliers were used in DSP slices for all FPGAs). 
As a conclusion for our smallest architecture, using large $w$ is not interesting. The reduction of the clock cycles count is canceled by the frequency drop for w68 or w136. Hence, the best solution is always w34 for A1 on all tested FPGAs.

\subsection{Architecture A2: CSWAP Optimization}

Architecture A2 is similar to A1 where we modified the CSWAP unit, version V2 (the architecture schematic is the same as Figure 2). The ML algorithm proposed in 24] uses one CSWAP operation at the end of each iteration and another CSWAP at the beginning of the next iteration with the same key bit operands. As there is no computation between these 2 consecutive CSWAP operations, we propose to merge them (this halves the calls to the CSWAP unit).

Our modified CSWAP-V2 uses 2 consecutive key bits: $k_{i}$ and $k_{i-1}$ (scalar $k$ is used starting MSB first). There is no swapping when $k_{i}=k_{i-1}$, and swapping when $k_{i} \neq k_{i-1}$ (we just need one xor gate). The very first CSWAP-V2 call is computed using bits 0 (current bit) and $k_{m-1}$ ("next" bit and MSB of $k$ ). The proposed modification does not change security aspects against SCAs. The accelerator is still constant-time and uniform. As for CSWAP in A1, we designed CSWAP-V2 with a uniform activity pipeline (see CSWAP description in Section 4.1).

Complete implementation results for A2 are reported in Table 7, A2 shows a similar behavior than $\mathrm{A} 1$ with respect to $w$ variations. A few elements can be noticed for A2 as summarized below:

- Clock cycles count in A2 is slightly smaller than A1 due to reduced number of CSWAP operations.

- Frequency is slightly higher for large $w$ compared to A1. Frequency variations are smaller in A2 than A1.

- Computation time in A2 is slightly smaller than A1: $-5-10 \%$ depending on the FPGA. The best solution is obtained for small $w$ (the $0.8 \%$ speed-up for w68/w136 in V5 is not relevant due to the large area increase).

- Area (LUTs, FFs and slices) in A2 is slightly smaller than A1 due to a simplified management of CSWAP operations: $-5-13 \%$ depending on the FPGA.

\begin{tabular}{|c|c||c|c|c|c|c|c|c|c|}
\hline FPGA & $\begin{array}{c}w \\
{[\mathrm{bit}]}\end{array}$ & LUT & FF & $\begin{array}{c}\text { logic } \\
\text { slices }\end{array}$ & $\begin{array}{c}\text { DSP } \\
\text { blocks }\end{array}$ & $\begin{array}{c}\text { RAM } \\
\text { blocks }\end{array}$ & $\begin{array}{c}\text { freq. } \\
{[\mathrm{MHz}]}\end{array}$ & $\begin{array}{c}\text { clock } \\
\text { cycles }\end{array}$ & $\begin{array}{c}\text { time } \\
{[\mathrm{ms}]}\end{array}$ \\
\hline \hline \multirow{3}{*}{ V4 } & 34 & 872 & 1624 & 1121 & 11 & 4 & 330 & 184,374 & 0.56 \\
\cline { 2 - 10 } & 68 & 1556 & 2637 & 1978 & 11 & 5 & 290 & 183,071 & 0.63 \\
\cline { 2 - 10 } & 136 & 2161 & 3027 & 2100 & 11 & 7 & 327 & 183,057 & 0.56 \\
\hline \multirow{3}{*}{ V5 } & 34 & 722 & 1605 & 541 & 11 & 4 & 360 & 184,374 & 0.51 \\
\cline { 2 - 10 } & 68 & 1196 & 2620 & 840 & 11 & 5 & 360 & 183,071 & 0.51 \\
\cline { 2 - 10 } & 136 & 1419 & 3009 & 944 & 11 & 7 & 360 & 183,057 & 0.51 \\
\hline \multirow{3}{*}{ S6 } & 34 & 940 & 1559 & 381 & 11 & 4 & 293 & 184,374 & 0.63 \\
\cline { 2 - 9 } & 68 & 1503 & 2565 & 553 & 11 & 5 & 262 & 183,071 & 0.70 \\
\cline { 2 - 9 } & 136 & 1890 & 2981 & 667 & 11 & 7 & 283 & 183,057 & 0.65 \\
\hline
\end{tabular}

Table 7. FPGA implementation results for architecture A2 (all BRAMs are $18 \mathrm{~Kb}$ ones, only $17 \times 17$ multipliers were used in DSP slices for all FPGAs). 


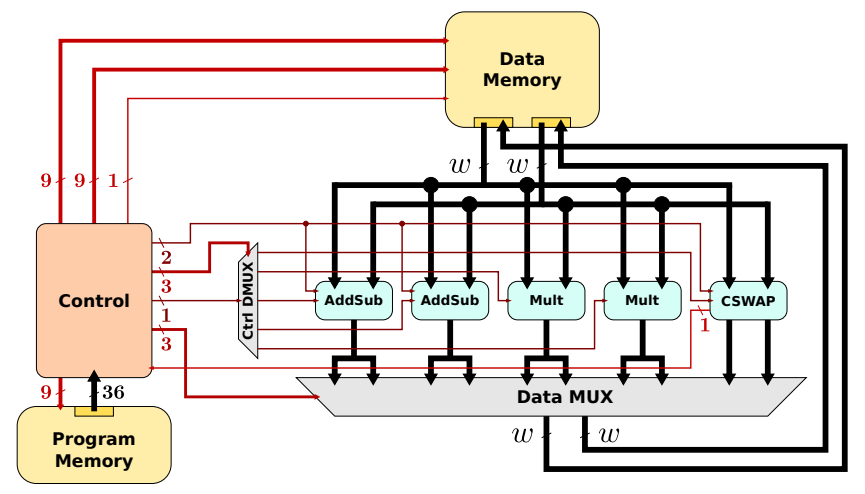

Fig. 3. Architecture A3 with its main units (arithmetic and memory), internal communication system, and control (warning: areas of boxes are not on a real scale).

- DSP slices and BRAMs are identical in A2 and A1 (not related to CSWAP-V2).

As a conclusion for architecture A2, the best configuration is always w34 for all tested FPGAs. This optimization is interesting since A2 is slightly more efficient than A1 in terms of both speed and area (about 10\%).

\subsection{Architecture A3: Large Architecture}

Architecture A3, depicted in Figure 3, embeds more arithmetic units: 2 AddSub and 2 Mult units. It can perform up to $6 \mathbb{F}_{p}$ multiplications in parallel (only 3 for A1/A2) using our hyper-threaded Mult unit (see Section 4.1 and 9]).

Complete implementation results for A3 are reported in Table 8. A3 behaves quite differently from A1/A2.

\begin{tabular}{|c|c||c|c|c|c|c|c|c|c|}
\hline FPGA & $\begin{array}{c}w \\
\text { [bit] }\end{array}$ & LUT & FF & $\begin{array}{l}\text { logic } \\
\text { slices }\end{array}$ & $\begin{array}{c}\text { DSP } \\
\text { blocks }\end{array}$ & $\begin{array}{c}\text { RAM } \\
\text { blocks }\end{array}$ & $\begin{array}{c}\text { freq. } \\
{[\text { MHz] }}\end{array}$ & $\begin{array}{c}\text { clock } \\
\text { cycles }\end{array}$ & $\begin{array}{c}\text { time } \\
{[\mathrm{ms}]}\end{array}$ \\
\hline \hline \multirow{3}{*}{ V4 } & 34 & 1462 & 2611 & 1783 & 22 & 6 & 294 & 188,218 & 0.64 \\
\cline { 2 - 10 } & 68 & 2802 & 4367 & 3468 & 22 & 7 & 282 & 124,191 & 0.44 \\
\cline { 2 - 10 } & 136 & 3768 & 5017 & 3660 & 22 & 9 & 285 & 119,057 & 0.42 \\
\hline \multirow{3}{*}{ V5 } & 34 & 1262 & 2607 & 921 & 22 & 6 & 358 & 188,218 & 0.53 \\
\cline { 2 - 9 } & 68 & 2290 & 4403 & 1409 & 22 & 7 & 345 & 124,191 & 0.36 \\
\cline { 2 - 9 } & 136 & 2737 & 4978 & 1594 & 22 & 9 & 348 & 119,057 & 0.34 \\
\hline \multirow{3}{*}{ S6 } & 34 & 1527 & 2503 & 668 & 22 & 6 & 265 & 188,218 & 0.71 \\
\cline { 2 - 9 } & 68 & 2421 & 4267 & 1020 & 22 & 7 & 225 & 124,191 & 0.55 \\
\cline { 2 - 9 } & 136 & 3007 & 4877 & 1131 & 22 & 9 & 225 & 119,057 & 0.53 \\
\hline
\end{tabular}

Table 8. FPGA implementation results for architecture A3 (all BRAMs are $18 \mathrm{~Kb}$ ones, only $17 \times 17$ multipliers were used in DSP slices for all FPGAs). 
- Adding 1 AddSub and 1 Mult increases LUTs by $60-90 \%$ depending on the FPGA and $w$. The second Mult adds 11 DSP slices and 2 BRAMs. This confirms that $\mathbb{F}_{p}$ units constitute the largest resources in the accelerator.

- Frequency is slightly smaller in A3 than A2 due to larger fanout and more complex control. The frequency drop for increasing $w$ values is very small for V4/V5 (less than $4 \%$ ), and about $15 \%$ for S6.

- Unlike A1/A2, $w$ has a large impact on the clock cycles count in A3: 34\% reduction for w68 compared to w34 and $36 \%$ for w136. More arithmetic operations in parallel put pressure on the memory and communication system. A larger $w$ allows to actually exploit more parallelism.

- Computation time benefits from the reduction of clock cycles count for large values of $w: 25$ to $35 \%$ reduction for w136 depending on the FPGA.

- A3 is faster than A2: from 16 to $35 \%$ depending on the FPGA. But this speed-up comes at the expense of a larger area.

As a conclusion for architecture A3, there is no best solution but various compromises in terms of area and speed. When area is limited, w34 is interesting but computation time is $25-33 \%$ larger. When speed is the main objective, w68 and w136 lead to the fastest solutions but the area overhead is important.

\subsection{Architecture A4: Clustered Architecture}

A closer look at Figure 1 shows that xDBLADD can be decomposed into 2 clusters of $\mathbb{F}_{p}$ operations with few dependencies using the red dashed horizontal line (i.e. one cluster above, one cluster below). Only 4 values have to be transferred from bottom cluster to top one at each ML iteration.

Architecture A4, depicted in Figure 4, was designed to exploit this decomposition using a clustered accelerator. It also embeds a new optimization of the CSWAP unit described below. To lighten Figure 4, control signals are not completely drawn but represented by small circles. Constants values are duplicated in each cluster memory when necessary (at no cost in BRAMs).

We added a new modification of the CSWAP behavior (V3). CSWAP operation is replaced by 2 new swapping operations $\mathrm{CS}_{0}$ and $\mathrm{CS}_{1}$ (with $4 \mathbb{F}_{p}$ operands):

- $\mathrm{CS}_{0}(A, B, C, D)$ returns $(A, B, C, B)$ if $k_{i}=0$, else it returns $(C, D, A, D)$

- $\operatorname{CS}_{1}(A, B, C, D)$ returns $(A, B, C, D)$ if $k_{i}=0$, else it returns $(C, D, A, B)$.

The modification of the CSWAP behavior (V3) was associated to a new schedule for xDBLADD presented in Figure 5. This figure is simplified, each black line now represents the communication of 4 operands from Figure 1. $\mathrm{H}$ box represents a set of $8 \mathbb{F}_{p}$ additions/subtractions, and $\mathrm{M}$ box a set of $4 \mathbb{F}_{p}$ multiplications (only 3 in the top right upper box of Figure 5 . $\mathrm{CS}_{0}$ and $\mathrm{CS}_{1}$ are respectively in charge of the first and last CSWAP-V3 of the original ML iteration. Square operations of the original xDBLADD have been replaced by multiplications in the new solution (i.e. $A^{2} \times B$ is now $A \times B \times A$ ) since we do not implement dedicated square units. This does not change the mathematical behavior nor the operations count compared to $\mathrm{A} 2 / \mathrm{A} 3$, but it allows to use $\mathrm{A} 4$ more efficiently. $\mathrm{CS}_{0}$ and $\mathrm{CS}_{1}$ operations also 


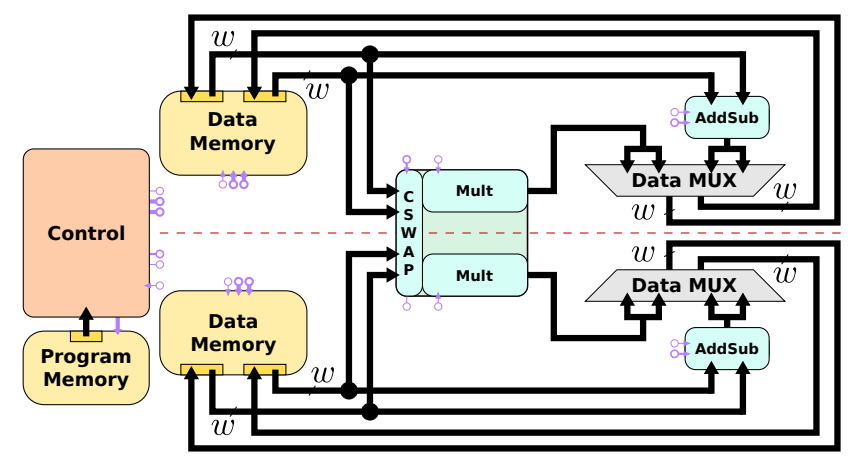

Fig. 4. Architecture A4 with its 2 clusters (units, data memory and local communication system), common control and new modification of CSWAP (warning: areas of boxes are not on a real scale).

act as "bridge" to exchange data between the 2 clusters when shared into a single CSWAP-V3 unit as illustrated in Figure 4 .

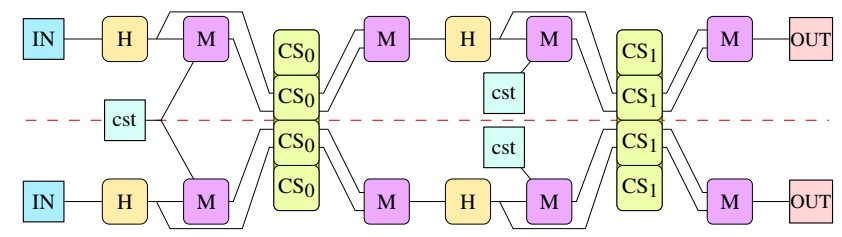

Fig. 5. Modified xDBLADD formula for architecture A4 with the new CSWAP behavior.

Architecture A4 (Figure 4) is based on two identical clusters. Each cluster contains: 1 AddSub, 1 Mult, 1 data memory and 1 local communication system. The shared CSWAP-V3 unit uses a 2-bit control mode to select the relevant swapping pattern according to the dependency graph in Figure 5. The control is shared for the 2 clusters, management of ML iterations and external inputs/outputs (at beginning/end of scalar multiplication).

Complete implementation results for A4 are reported in Table 9 A few elements are summarized below:

- Clock cycles count is reduced for w34 but slightly increased for w68 and w136 compared to A3. This is due to additional constraints on the scheduler for A4 since all units do not share a common memory and clusters can only exchange data during the new modified CSWAP-V3.

- Frequency is higher in A4 compared to A3 in most of case (and very similar in the other cases). This is due to a more local control and a smaller fanout (most of signals are local to each cluster). 


\begin{tabular}{|c|c|c|c|c|c|c|c|c|c|}
\hline FPGA & $\begin{array}{c}w \\
\text { [bit] }\end{array}$ & LUT & FF & $\begin{array}{l}\text { logic } \\
\text { slices }\end{array}$ & $\begin{array}{c}\text { DSP } \\
\text { blocks }\end{array}$ & $\begin{array}{c}\text { RAM } \\
\text { blocks }\end{array}$ & $\begin{array}{c}\text { freq. } \\
\text { [MHz] }\end{array}$ & $\begin{array}{c}\text { clock } \\
\text { cycles }\end{array}$ & $\begin{array}{c}\text { time } \\
\text { [ms] }\end{array}$ \\
\hline \hline \multirow{3}{*}{ V4 } & 34 & 1695 & 2950 & 2158 & 22 & 7 & 324 & 142,119 & 0.44 \\
\cline { 2 - 10 } & 68 & 2804 & 4282 & 3184 & 22 & 9 & 290 & 128,021 & 0.44 \\
\cline { 2 - 10 } & 136 & 3171 & 4994 & 3337 & 22 & 13 & 299 & 125,456 & 0.42 \\
\hline \multirow{3}{*}{ V5 } & 34 & 1370 & 2953 & 1013 & 22 & 7 & 358 & 142,119 & 0.40 \\
\cline { 2 - 10 } & 68 & 2095 & 4259 & 1358 & 22 & 9 & 337 & 128,021 & 0.38 \\
\cline { 2 - 9 } & 136 & 2514 & 4952 & 1589 & 22 & 13 & 313 & 125,456 & 0.40 \\
\hline \multirow{3}{*}{ S6 } & 34 & 1564 & 2089 & 758 & 22 & 7 & 262 & 142,119 & 0.54 \\
\cline { 2 - 9 } & 68 & 2387 & 4030 & 1060 & 22 & 9 & 239 & 128,021 & 0.54 \\
\cline { 2 - 9 } & 136 & 3181 & 4786 & 1136 & 22 & 13 & 251 & 125,456 & 0.50 \\
\hline
\end{tabular}

Table 9. FPGA implementation results for architecture A4 (all BRAMs are $18 \mathrm{~Kb}$ ones, only $17 \times 17$ multipliers were used in DSP slices for all FPGAs).

- Computation time is significantly reduced for small $w$ values. For instance, w34 leads to a similar speed than A3 but with much smaller architecture.

- On V5, the fastest solution is the intermediate configuration w68.

- DSP slices amount is exactly the same in A4 and A3 (both use 2 Mult units).

- BRAMs amount is larger in A4 than A3 due to the 2 local memories (one in each cluster). The number of BRAMs increases with $w$ accordingly to Table 2 configurations.

- Area results in terms of LUTs are quite different from previous architectures. It increases for w34 (up to $+15 \%$ ) but decreases for w136 (up to $-16 \%$ ) compared to A3.

- Adding a second memory unit allows parallel read/write accesses and helps to quickly extract more parallel operations.

As a conclusion for architecture A4, selecting the right set of parameters depends a lot on the objective (high speed or low cost) and the FPGA family. When the main objective is selecting the absolute smallest accelerator, A4 is less interesting then A3. When the main objective is selecting the absolute fastest accelerator, A4 is interesting for low-cost S6 FPGA (but A3 is better for V4/V5). But in practice, A4 is interesting for accelerators almost as fast as the fastest A3 but for much smaller area. For instance on V4, w34 configuration is only $5 \%$ slower than the absolute fastest A3 solution with an area reduced by $55 \%$ in LUTs and $22 \%$ in BRAMs.

\section{Comparisons}

Figure 6 reports all the implementation results for the 4 proposed architectures and $w$ configurations on 3 different FPGAs. This figure only reports LUTs for area since there are only a very few different numbers of BRAMs and DSP slices given in Table 10. The best configuration (architecture type, $w$ ) depends on the objective (high speed or low area) and target FPGA. Then exploration tools at architecture level are helpful for designers and users. For low-area solution, 


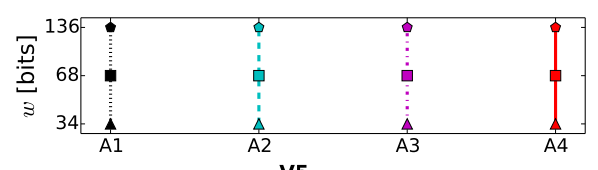

V4
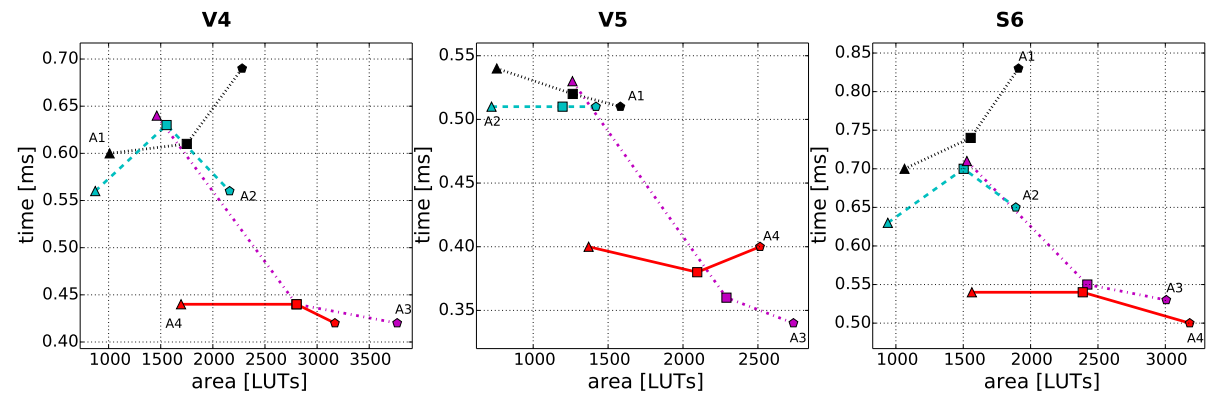

Fig. 6. Trade-offs for our architectures A1-4 in terms of area (LUTs) and computation time for all $w$ configurations and FPGAs (legend is top figure).

\begin{tabular}{|c|c|c|c|c|c|c|c|}
\hline archi. & $\begin{array}{c}w \\
\text { [bit] }\end{array}$ & target & $\begin{array}{l}\text { logic } \\
\text { slices }\end{array}$ & $\begin{array}{c}\text { DSP } \\
\text { blocks }\end{array}$ & $\begin{array}{l}\text { RAM } \\
\text { blocks }\end{array}$ & $\begin{array}{c}\text { freq. } \\
{[\mathrm{MHz}]}\end{array}$ & $\begin{array}{l}\text { time } \\
{[\mathrm{ms}]}\end{array}$ \\
\hline$\overline{\mathrm{A} 2}$ & $\overline{34}$ & \multirow{3}{*}{ V4 } & 1121 & $\overline{11}$ & 4 & 330 & 0.56 \\
\hline A3 & 136 & & 3660 & 22 & 9 & 285 & 0.42 \\
\hline $\mathrm{A} 4$ & 34 & & 2158 & 22 & 7 & 324 & 0.44 \\
\hline A2 & 34 & \multirow{3}{*}{ V5 } & 541 & 11 & 4 & 360 & 0.51 \\
\hline A3 & 136 & & 1594 & 22 & 9 & 348 & 0.34 \\
\hline A4 & 34 & & 1013 & 22 & 7 & 358 & 0.40 \\
\hline $\mathrm{A} 2$ & 34 & \multirow{3}{*}{ S6 } & 381 & 11 & 4 & 293 & 0.63 \\
\hline$\overline{\mathrm{A} 3}$ & 136 & & 1131 & 22 & 9 & 225 & 0.53 \\
\hline $\mathrm{A} 4$ & 34 & & 758 & 22 & 7 & 262 & 0.54 \\
\hline
\end{tabular}

Table 10. Summary of our most interesting FPGA implementation results (those on Pareto front from Figure 60.

A2 is always the best one. For high speed, A4 is a very good cost-performance trade-off on V4 and S6 (A3 is the fastest on V5 but with a large area).

Most of hardware HECC implementations use curves over $\mathbb{F}_{2^{n}}$ with low security levels (typically $81-89$ bits fields). Table 11 reports some of them. None of those HECC implementations embed hardware level protections against SCAs. Some of them use algorithmic protections for scalar multiplication such as the Montgomery ladder. Our $\mathbb{F}_{p}$ accelerators show similar computation times but for a much higher security level (128 bits) on more recent FPGAs. To the best of our knowledge, we found only one hardware implementation of HECC over $\mathbb{F}_{p}$ in [1. It is an $0.13 \mu \mathrm{m}$ ASIC implementation for 81-bit generic prime $p$ with $502.8 \mathrm{~ms}$ computation time for scalar multiplication at $1 \mathrm{MHz}$. The very low reported frequency makes comparisons quite difficult. 


\begin{tabular}{|c|c|c|c|c|c|c|c|c|c|}
\hline ref. & year & target & $n$ & LUT & $\mathrm{FF}$ & $\begin{array}{l}\text { logic } \\
\text { slices }\end{array}$ & $\begin{array}{l}\text { RAM } \\
\text { blocks }\end{array}$ & $\begin{array}{l}\text { freq. } \\
{[\mathrm{MHz}]}\end{array}$ & $\begin{array}{l}\text { time } \\
{[\mathrm{ms}]}\end{array}$ \\
\hline 3 & 2006 & Virtex 2 Pro & 83 & 20999 & n.a. & 11296 & n.a. & 166 & 0.5 \\
\hline 8 & 2008 & $\mathrm{XC} 2 \mathrm{~V} 4000$ & 83 & n.a. & n.a. & 2316 & 6 & 125 & 0.31 \\
\hline \multirow{2}{*}{13} & \multirow{2}{*}{2004} & $\mathrm{XC2V4000}$ & 89 & 8451 & 2178 & 4995 & 1 & 54 & 1.02 \\
\hline & & XC2V4000 & 89 & 16459 & 4437 & 9950 & 0 & 57 & 0.44 \\
\hline \multirow{2}{*}{25} & \multirow{2}{*}{2006} & Virtex 2 Pro & 83 & n.a. & n.a. & 2446 & $1^{*}$ & 100 & 0.99 \\
\hline & & Virtex 2 Pro & 83 & n.a. & n.a. & 6586 & $3^{*}$ & 100 & 0.42 \\
\hline \multirow{2}{*}{26} & \multirow{2}{*}{2016} & Virtex 2 & 83 & n.a. & n.a. & 5734 & n.a. & 145 & 0.3 \\
\hline & & XC5V240 & 83 & n.a. & n.a. & 5086 & n.a. & 175 & 0.29 \\
\hline \multirow{4}{*}{27} & \multirow{4}{*}{2004} & Virtex 2 Pro & 81 & n.a. & n.a. & 4039 & 1 & 57 & 0.79 \\
\hline & & Virtex 2 Pro & 81 & n.a. & n.a. & 7737 & 0 & 61 & 0.39 \\
\hline & & $\mathrm{XC} 2 \mathrm{~V} 4000$ & 81 & n.a. & n.a. & 3955 & 1 & 54 & 0.83 \\
\hline & & $\mathrm{XC2V4000}$ & 81 & n.a. & n.a. & 7785 & n.a. & 57 & 0.42 \\
\hline 7 & 2007 & $\mathrm{XC} 2 \mathrm{~V} 8000$ & 113 & n.a. & n.a. & 25271 & n.a. & 45 & 2.03 \\
\hline
\end{tabular}

Table 11. FPGA implementation results for various HECC solutions over $\mathbb{F}_{2^{n}}$ from state of the art (warning: security levels are much lower than our solutions). For $\mathbb{F}_{2^{n}}$ DSP slices cannot be used. Values with a "** are estimated number of RAM blocks based on paper explanations.

\begin{tabular}{|c|c|c|c|c|c|c|c|c|c|c|}
\hline ref. & year & target & $p$ & LUT & $\mathrm{FF}$ & $\begin{array}{l}\text { logic } \\
\text { slices }\end{array}$ & $\begin{array}{c}\text { DSP } \\
\text { blocks }\end{array}$ & $\begin{array}{l}\text { RAM } \\
\text { blocks }\end{array}$ & $\begin{array}{c}\text { freq. } \\
{[\mathrm{MHz}]}\end{array}$ & $\begin{array}{l}\text { time } \\
{[\mathrm{ms}]}\end{array}$ \\
\hline 2 & 2014 & XCV6FX760 & NIST-256 & 32900 & n.a. & 11200 & 289 & 128 & 100 & 0.4 \\
\hline \multirow{2}{*}{11} & \multirow{2}{*}{2008} & XC4VFX12 & NIST-256 & 2589 & 2028 & 1715 & 32 & 11 & 490 & 0.5 \\
\hline & & XC4VFX12 & NIST-256 & 34896 & 32430 & 24574 & 512 & 176 & 375 & 0.04 \\
\hline \multirow{2}{*}{17} & \multirow{2}{*}{2012} & XC4VFX12 & GEN-256 & n.a. & n.a. & 2901 & 14 & n.a. & 227 & 1.09 \\
\hline & & XC5VLX110 & GEN-256 & n.a. & n.a. & 3657 & 10 & n.a. & 263 & 0.86 \\
\hline \multirow{2}{*}{19} & \multirow{2}{*}{2013} & XC4VLX100 & GEN-256 & 5740 & 4876 & 4655 & 37 & 11 & 250 & 0.44 \\
\hline & & XC5LX110T & GEN-256 & 4177 & 4792 & 1725 & 37 & 10 & 291 & 0.38 \\
\hline
\end{tabular}

Table 12. FPGA implementation results for various ECC solutions over $\mathbb{F}_{p}$ and 128-bit security level from state of the art.

Directly comparing our accelerators with implementations of HECC over $\mathbb{F}_{2^{n}}$ for much lower security level is not possible. Then, in Table 12, we report some of the best FPGA implementations results we found in the state of the art for ECC solutions over $\mathbb{F}_{p}$ and 128 bits security level. In practice using hundred of DSP blocks and BRAMs may not be a realistic solution for embedded systems. In [2] several SCAs protections have been presented: DBL\&ADD-always, ML, scalar randomization, units with uniform behavior, randomization of memory addresses and noise addition. Those protections impact the number of logic slices but not those of DSPs and BRAMs (very huge is this work).

Compared to [19, a very optimized fast and compact solution from state of the art using randomized Jacobian coordinates, our accelerator have a very similar computation time $(0.44 \mathrm{~ms})$ but with $40 \%$ reduction in DSP and RAM blocks and $53 \%$ reduction of logic slices on V4 FPGA (similar for V5). 


\section{Conclusion and Future Prospects}

We proposed the first hardware implementation of Kummer based HECC solution for 128-bit security level. Various architectures and parameters have been explored using in-house tools. Several architectures with different amount of internal parallelism have been optimized and fully implemented on 3 different FPGAs. The obtained results lead to similar speed than the best curve based solutions for embedded systems but with an area almost divided by 2 (-40\% for DSP and RAM blocks and $-60 \%$ for logic slices). Those results were obtained with generic prime fields and fully programmable architectures (which is not the case in most of state of the art implementations).

In the future, we plan to optimize our tools and architectures, evaluate the security against SCAs using real measurement setup, automate the control generation of the accelerator, and publish our architectures as open source hardware.

\section{Acknowledgment}

This work was done in the HAH project http://h-a-h.inria.fr/ partially funded by Labex CominLab, Labex Lebesgue and Brittany Region.

\section{References}

1. H.-R. Ahmadi, A. Afzali-Kusha, M. Pedram, and M. Mosaffa. Flexible prime-field genus 2 hyperelliptic curve cryptography processor with low power consumption and uniform power draw. ETRI journal, 37(1):107-117, February 2015.

2. H. Alrimeih and D. Rakhmatov. Fast and flexible hardware support for ECC over multiple standard prime fields. IEEE Transactions on Very Large Scale Integration (VLSI) Systems, 22(12):2661-2674, January 2014.

3. L. Batina, N. Mentens, B. Preneel, and I. Verbauwhede. Flexible hardware architectures for curve-based cryptography. In Proc. IEEE International Symposium on Circuits and Systems (ISCAS), pages 4839-4842. IEEE, May 2006.

4. D. J. Bernstein and T. Lange. Explicit-formulas database.http://hyperelliptic. org/EFD/

5. J. W. Bos, C. Costello, H. Hisil, and K. Lauter. Fast cryptography in genus 2. Journal of Cryptology, 29(1):28-60, January 2016.

6. H. Cohen and G. Frey, editors. Handbook of Elliptic and Hyperelliptic Curve Cryptography. Discrete Maths and Applications. Chapman \& Hall/CRC, July 2005.

7. G. Elias, A. Miri, and T.-H. Yeap. On efficient implementation of FPGA-based hyperelliptic curve cryptosystems. Computers \& Electrical Engineering, 33(5):349366, July 2007.

8. J. Fan, L. Batina, and I. Verbauwhede. HECC goes embedded: an area-efficient implementation of HECC. In Proc. 15th Workshop on Selected Areas in Cryptography $(S A C)$, volume 5381 of $L N C S$, pages 387-400. Springer, August 2008.

9. G. Gallin and A. Tisserand. Hyper-threaded multiplier for HECC. In Proc. 51st Asilomar Conference on Signals, Systems and Computers, Pacific Grove, CA, USA, October 2017. IEEE. 
10. P. Gaudry. Fast genus 2 arithmetic based on theta functions. Journal of Mathematical Cryptology, 1(3):243-265, August 2007.

11. T. Güneysu and C. Paar. Ultra high performance ECC over NIST primes on commercial FPGAs. In Proc. 10th Conf. Cryptographic Hardware and Embedded Systems (CHES), volume 5154 of LNCS, pages 62-78. Springer, August 2008.

12. D. Hankerson, A. Menezes, and S. Vanstone. Guide to Elliptic Curve Cryptography. Springer, 2004.

13. H. Kim, T. J. Wollinger, Y. Choi, K. Chung, and C. Paar. Hyperelliptic curve coprocessors on a FPGA. In Proc. 5th International Workshop on Information Security Applications (WISA), volume 3325 of $L N C S$, pages 360-374. Springer, August 2004.

14. N. Koblitz. Hyperelliptic cryptosystems. Journal of Cryptology, 1(3):139-150, October 1989.

15. C. K. Koc, T. Acar, and B. S. Kaliski. Analyzing and comparing Montgomery multiplication algorithms. IEEE Micro, 16(3):26-33, June 1996.

16. Satoh Lab. and Morita Tech. Side-channel attack user reference architecture (SAKURA), 2013.

17. J.-Y. Lai, Y.-S. Wang, and C.-T. Huang. High-performance architecture for elliptic curve cryptography over prime fields on FPGAs. Interdisciplinary Information Sciences, 18(2):167-173, 2012.

18. T. Lange. Formulae for arithmetic on genus 2 hyperelliptic curves. Applicable Algebra in Eng., Communication and Computing, 15(5):295-328, February 2005.

19. Y. Ma, Z. Liu, W. Pan, and J. Jing. A high-speed elliptic curve cryptographic processor for generic curves over $\mathrm{GF}(p)$. In Proc. 20th International Workshop on Selected Areas in Cryptography (SAC), volume 8282 of LNCS, pages 421-437, Burnaby, BC, Canada, August 2013. Springer.

20. S. Mangard, E. Oswald, and T. Popp. Power Analysis Attacks: Revealing the Secrets of Smart Cards. Springer, 2007.

21. P. L. Montgomery. Modular multiplication without trial division. Mathematics of Computation, 44(170):519-521, April 1985.

22. P. L. Montgomery. Speeding the pollar and elliptic curves methods of factorisation. Mathematics of Computation, 48(177):243-264, January 1987.

23. H. Orup. Simplifying quotient determination in high-radix modular multiplication. In Proc. 12th IEEE Symposium on Computer Arithmetic (ARITH), pages 193-199, Bath, UK, July 1995. IEEE.

24. J. Renes, P. Schwabe, B. Smith, and L. Batina. $\mu$ Kummer: Efficient hyperelliptic signatures and key exchange on microcontrollers. In B. Gierlichs and A. Y. Poschmann, editors, Proc. 18th International Conference on Cryptographic Hardware and Embedded Systems (CHES), volume 9813 of LNCS, pages 301-320, Santa Barbara, CA, USA, August 2016. Springer.

25. K. Sakiyama, L. Batina, B. Preneel, and I. Verbauwhede. Superscalar coprocessor for high-speed curve-based cryptography. In Proc. 8th Workshop on Cryptographic Hardware and Embedded Systems (CHES), volume 4249 of LNCS, pages 415-429. Springer, October 2006.

26. A. Sghaier, C. Massoud, M. Zeghid, and M. Machhout. Flexible hardware implementation of hyperelliptic curves cryptosystem. International Journal of Computer Science and Information Security (IJCSIS), 14(4), April 2016.

27. T. Wollinger. Software and hardware implementation of hyperelliptic curve cryptosystems. Ruhr University Bochum, 2004. 